\title{
Sequencing and Integration of Cervical Manual Therapy and Vestibulo-oculomotor Therapy for Concussion Symptoms: Retrospective Analysis
}

\author{
Christopher Kevin Wong, PT, PhD, OCS ${ }^{1}$, Lauren Ziaks, PT, DPT, ATC ${ }^{2}$, Samantha Vargas, PT, DPT ${ }^{3}$, Tessia DeMattos, \\ PT, DPT ${ }^{3}$, Chelsea Brown, PT, DPT ${ }^{2}$ \\ ${ }^{1}$ Department of Rehabilitation \& Regenerative Medicine, Columbia University Irving Medical Center, ${ }^{2}$ Park City Hospital, Intermountain Healthcare, ${ }^{3}$ \\ Vagelos College of Physicians \& Surgeons, Columbia University \\ Keywords: vestibular rehabilitation, vision therapy, manual therapy, cervicogenic headache, concussion \\ https://doi.org/10.26603/001c.18825
}

\section{International Journal of Sports Physical Therapy}

Vol. 16, Issue 1, 2021

\begin{abstract}
Background
After concussion many people have cervicogenic headache, visual dysfunction, and vestibular deficits that can be attributed to brain injury, cervical injury, or both. While clinical practice guidelines outline treatments to address the symptoms that arise from the multiple involved systems, no preferred treatment sequence for post-concussion syndrome has emerged.
\end{abstract}

\section{Purpose}

This study sought to describe the clinical and patient-reported outcomes for people with post-concussion symptoms after a protocol sequenced to address cervical dysfunction and benign paroxysmal positional vertigo within the first three weeks of injury, followed by integrated vision and vestibular therapy.

\section{Study Design}

Retrospective longitudinal cohort analysis

\section{Methods}

Records from a concussion clinic for 38 patients (25 male 13 female, aged $26.9 \pm 19.7$ years) with post-concussion symptoms due to sports, falls, assaults, and motor vehicle accident injuries were analyzed. Musculoskeletal, vision, and vestibular system functions were assessed after pragmatic treatment including early cervical manual therapy and canalith repositioning treatment-when indicated-integrated with advanced vision and vestibular rehabilitation. Patient-reported outcomes included the Post-Concussion Symptom Scale (PCSS) for general symptoms; and for specific symptoms, the Dizziness Handicap Index (DHI), Convergence Insufficiency Symptom Scale (CISS), Activities-specific Balance Confidence scale (ABC), and the Brain Injury Vision Symptom Survey (BIVSS). Paired t-tests with Bonferroni correction to minimize familywise error $(\mathrm{p}<0.05)$ were used to analyze the clinical and patient-reported outcomes.

\section{Results}

After $10.4 \pm 4.8$ sessions over $57.6 \pm 34.0$ days, general symptoms improved on the PCSS $(\mathrm{p}=0.001,95 \% \mathrm{CI}=12.4-30.6)$; and specific symptoms on the DHI $(\mathrm{p}<0.001$, 95\%CI=14.5-33.2), CISS ( $<<0.002,95 \% \mathrm{CI}=7.1-18.3), \mathrm{ABC}(\mathrm{p}<0.024,95 \% \mathrm{CI}=-.3--.1)$, and BIVSS ( $\mathrm{p}<0.001,95 \% \mathrm{CI}=13.4-28.0)$. Clinical measures improved including cervical range-of-motion (55.6\% fully restored), benign paroxysmal positional vertigo symptoms

\footnotetext{
a Corresponding author:

Christopher Kevin Wong, PT, PhD, OCS

Department of Rehabilitation \& Regenerative Medicine

Columbia University Irving Medical Center

617 West 168th Street, New York, NY 10032
} 
(28/28, fully resolved), Brock string visual convergence $(\mathrm{p}<0.001,95 \% \mathrm{CI}=3.3-6.3)$, and score on the Balance Error Scoring System ( $\mathrm{p}<0.001,95 \% \mathrm{CI}=5.5-11.6)$.

\section{Conclusion}

A rehabilitation approach for post-concussion syndrome that sequenced cervical dysfunction and benign paroxysmal positional vertigo treatment within the first three weeks of injury followed by integrated vision and vestibular therapy improved clinical and patient-reported outcomes.

\section{Level of Evidence: $2 b$}

\section{INTRODUCTION}

Sport and recreation-related concussion is a rapidly growing problem, with an estimated 1.6-3.8 million people affected per year. ${ }^{1}$ Concussion also affects people involved in transportation accidents, fall-related injuries, and other trauma. ${ }^{1}$ Defined as a mild traumatic brain injury induced by direct or indirect biomechanical forces, concussion may or may not include loss of consciousness. ${ }^{2}$ While more than $80 \%$ of adults recover from sports-related concussions in 7-10 days, concussive symptoms can persist much longer and take weeks or months to resolve after injury. ${ }^{3,4}$ Symptoms lasting longer than the generally accepted three-week period can be referred to as protracted concussion recovery, ${ }^{3}$ a particular problem among adolescent athletes..$^{5}$ After concussion in adolescents, protracted recovery is common with most reporting symptoms lasting more than a month: boys averaging seven weeks and girls an even longer 10 weeks. $^{5}$

The signs and symptoms observed in protracted concussion recovery cannot always be linked to abnormal brain imaging or neuropathological changes, but rather are often attributed to multiple physiologic systems. ${ }^{6}$ Typical findings involve the musculoskeletal, visual, vestibular, cardiovascular, and autonomic systems. ${ }^{4}$ Signs and symptoms after concussion attributed to the musculoskeletal system are commonly localized to the cervical region and include joint and myofascial hypomobility that limits range-of-motion $(\mathrm{ROM})^{7}$ and cervicogenic headaches. ${ }^{6}$ Cervicogenic headaches are secondary headaches encompassing neck or facial pain associated with bony, myofascial, and disc disorders of the cervical spine. ${ }^{8}$ Cervicogenic headaches develop with the onset of cervical disorders, such as biomechanical forces occurring during mild traumatic brain injury, and significantly improve with the resolution of cervical disorders. ${ }^{8}$ Visual or oculomotor symptoms are not limited to but commonly include blurred vision, double vision, difficulty concentrating, and difficulty with activities such as reading. ${ }^{3}$ Vestibular system symptoms include dizziness, nausea, fatigue, balance difficulties, and blurry vision with head movements known as oscillopsia. ${ }^{9}$ In addition, final clinical recovery can be delayed by dysautonomia that can decrease exercise tolerance and limit return to sport; ${ }^{3,4}$ or cognitive and psychological issues that impair return to learn or work. ${ }^{10}$

Many post-concussion symptoms can be attributed to brain injury, cervical spine injury, or both, ${ }^{3,4}$ and can have a long lasting negative impact on function, but can be modified through treatment of the relevant physiologic systems. ${ }^{3}$ Medical and athletic management of concussions has been delineated in a recent clinical practice guideline. ${ }^{10}$ In 2020, the American Physical Therapy Association published a clinical practice guideline describing current best practice for physical therapy care of concussion including cervical musculoskeletal, vestibulo-oculomotor, autonomic/exertional tolerance, motor function, and psychological and sociological impairments. ${ }^{11}$ Manual therapy may provide added value to the benefits of exercise in reducing cervicogenic headache and associated neck pain and dysfunction. ${ }^{12}$ For instance, treatments including cervical muscle active trigger point release and manipulative therapy can reduce intensity, frequency, and cervicogenic headache symptom duration. ${ }^{13}$ Visual system impairment can be reduced with vision therapy including oculomotor and vergence training using targets with numbers and letters, and fusion exercises such as stereograms and tranaglyphs. ${ }^{14}$ Vestibular therapy such as vestibulo-ocular reflex movements for adaptation, habituation exercises, and substitution including static and dynamic balance exercises can improve vestibulo-oculomotor symptoms after concussions. 9,15 Although multisystem involvement is common, existing treatment protocols typically describe interventions focused on the symptom clusters arising from one system or another. ${ }^{9}$

Treatment outcomes for post-concussion syndrome can be complicated by the interconnectedness of the vestibular, visual, and musculoskeletal systems. ${ }^{16}$ For instance, vestibular therapy requires the ability to maintain visual fixation on a point while turning the head. ${ }^{9}$ Furthermore, deficits in cervical spine and oculomotor coordination can lead to dizziness and blurred vision. ${ }^{17}$ The function of the cervical spine, visual and vestibular systems are not isolated. Without the ability to have both eyes converge on a point or comfortably rotate the cervical spine, compensations can occur. ${ }^{9}$ While current clinical practice guidelines outline potential treatments to help address the symptoms that occur in each of the involved systems, no preferred treatment sequence for patients with post-concussion syndrome has emerged. ${ }^{10,11}$ The purpose of this study was to describe the clinical and patient-reported outcomes for people with post-concussion symptoms after a rehabilitation protocol sequenced to address cervical dysfunction and benign paroxysmal positional vertigo within the first three weeks of injury, followed by integrated vision and vestibular therapy. 
Table 1: Characteristics of the Patient Cohort

\begin{tabular}{|l|c|}
\hline Sex & 25 males, 13 female \\
\hline Age & $26.9 \pm 19.7$ years \\
\hline History of Concussion & $39.5 \%$ \\
\hline Activity at Time of Injury & $60.5 \%$ \\
Sport & $18.4 \%$ \\
Fall & $13.2 \%$ \\
Motor Vehicle Accident & $7.9 \%$ \\
Assault & $26.3 \%$ \\
\hline Symptoms & $10.5 \%$ \\
Vision and Vestibular only & $63.2 \%$ \\
Musculoskeletal (includes cervicogenic headache) only & \\
Vision and Vestibular with Musculoskeletal & \\
\hline
\end{tabular}

\section{METHOD}

\section{DESIGN}

This study was a retrospective longitudinal cohort analysis of pre-existing data obtained from August 2016 - March 2017 from a single concussion clinic. Deidentified coded data was received for analysis from the participating concussion clinic in accordance with the protocol approved by the Columbia University Irving Medical Center Institutional Review Board of the participating University Medical Center.

\section{SAMPLE}

The records of 59 people with post-concussion symptoms were reviewed. The 38 patients (25 male and 13 female, aged $26.9 \pm 19.7$ years) who had both evaluation and re-evaluation data-follow-up progress report or discharge assessment-were included for analysis. All patients had been referred for symptoms post-concussion and suspected mild traumatic brain injury with cervicogenic, visual, and/or vestibular symptoms. The average time from injury to initial evaluation was $31.6 \pm 50.4$ days, with 17 reporting protracted symptoms beyond 21 days. The most common presentation was mixed cervical, vision and vestibular symptoms reported by 24 (63.2\%); followed by 10 (26.3\%) that had vision and vestibular symptoms; and $4(10.5 \%)$ that reported only cervicogenic symptoms including headache. Concussion etiology was varied with concussions occurring after sport (60.5\%), fall (18.4\%), motor vehicle accident (13.2\%), and assaults (7.9\%). A history of prior concussion was reported by 15 (39.5\%). Table 1 .

\section{ASSESSMENTS}

Clinical findings and patient-reported outcomes were obtained by one trained clinician in the participating specialized concussion rehabilitation clinic. To minimize variation among different clinicians for patients who had limited cervical ROM at the initial evaluation, the cervical ROM measure was reduced for analysis in this study to a simple dichotomous outcome. ROM was either normal, denoted in the clinical notes as normal, within normal or within functional limits, or limited, defined as any limitation recorded in degrees, percentage, or qualitative term. Visual system outcome measures included the Brock string score for convergence and divergence distance, ${ }^{18}$ the cover-uncover test to screen for malalignment and strabismus that would require neuro-optometry referral, ${ }^{19}$ and clinical screening for quality and speed of smooth pursuits and saccades, and near point convergence. ${ }^{20}$ Vestibular measures included Dix-Hallpike and Supine Roll Tests for benign paroxysmal positional vertigo (BPPV). Additional vestibular screening assessments included vestibular ocular reflex, visual motion sensitivity and the Balance Error Scoring System (BESS), which has been shown to be a reliable measure in athletes post-concussion. ${ }^{21}$

Patient-reported outcomes included five scales that collectively provide insight on the potential symptoms that stem from deficits in the cervical spine, vestibular and visual systems. ${ }^{22-26}$ The Post-Concussion Symptom Scale (PCSS) assesses general post-concussion symptoms with specific symptom indices for associated sequelae including headache, with variable reliability for the different indices. ${ }^{22}$ The Dizziness Handicap Inventory (DHI) assesses the impact of dizziness on functional, emotional, and physical quality of life with excellent reliability and internal consistency. ${ }^{23}$ Visual impairment was assessed with the Convergence Insufficiency Symptom Survey (CISS), which has excellent reliability, ${ }^{24}$ and the Acquired Traumatic Brain Injury Vision Symptom Questionnaire, since adapted and renamed the Brain Injury Vision Symptom Survey (BIVSS). ${ }^{25}$ Finally, the Activities-specific Balance Confidence (ABC) scale assesses individual sense of balance and correlates with cognitive measures after concussion. ${ }^{26}$ See Table 2.

\section{INTERVENTIONS}

Patients received treatment integrating cervical, vision and vestibular dysfunction as indicated by individual impairment and determined pragmatically by the trained concussion clinic treatment specialist, as previously outlined in a treatment algorithm. ${ }^{27}$ Patients evaluated within the threeweek post-injury recovery window began treatment for cervical dysfunction and symptoms. Patients evaluated more than three weeks post-injury, consistent with protracted concussion recovery, ${ }^{3}$ began with treatment for cervical dysfunction integrated with vision and vestibular therapy. Because resolution of the multiple systems varied, treat- 
Table 2: Clinical and Patient-reported Outcomes

\begin{tabular}{|c|c|c|}
\hline System & Assessments & Outcomes \\
\hline Combined & Post-Concussion Symptom Scale & $p=0.001,95 \% C l 12.4-30.6, n=19, d=1.18$ \\
\hline Musculoskeletal & Cervical ROM & $55.6 \%(15 / 27)$ regained full ROM \\
\hline $\begin{array}{l}\text { Vision } \\
\text { Convergence } \\
\text { Saccades }\end{array}$ & $\begin{array}{l}\text { Convergence Insufficiency Scale } \\
\text { Brain Injury Vision Symptom Survey } \\
\text { Near-point Brock string score } \\
\text { Saccade score }\end{array}$ & $\begin{array}{l}p=0.002,95 \% C l 7.1-18.3, n=19, d=1.04 \\
p<0.001,95 \% C l=13.4-28.0, n=15, d=1.21 \\
p<0.001,95 \% C l 3.3-6.3, n=23, d=1.20 \\
p>0.05, n=13\end{array}$ \\
\hline $\begin{array}{l}\text { Vestibular } \\
\text { BPPV } \\
\text { Balance-objective } \\
\text { Balance-subjective }\end{array}$ & $\begin{array}{l}\text { Dizziness Handicap Index } \\
\text { BPPV symptoms } \\
\text { Balance Error Scoring System } \\
\text { Activity-specific Balance Confidence }\end{array}$ & $\begin{array}{l}p<0.001,95 \% \mathrm{Cl} 14.5-33.2, n=19, d=1.04 \\
100 \%(28 / 28) \text { resolved fully } \\
p<0.001,95 \% \mathrm{Cl} 5.5-11.6, n=14, d=0.99 \\
p=0.024,95 \% \mathrm{Cl}-0.3--0.1, n=16, d=-0.89\end{array}$ \\
\hline
\end{tabular}

BPPV = Benign Paroxysmal Positional Vertigo

ROM = Range of Motion

95\%CI $=95 \%$ Confidence Interval

ments overlapped.

Cervical dysfunction was treated with manual therapy and exercise, ${ }^{12}$ followed by canalith repositioning procedure for BPPV when present. ${ }^{28}$ Vision therapy included vision exercises for smooth pursuits, saccades, complex motor tasks including divided attention and laterality, and vergences. $^{29}$ Vestibular rehabilitation therapy was integrated for substitution (dynamic balance exercises with visual restriction and on compliant surfaces), vestibular ocular reflex training for gaze stability to address adaptation and exercises for habituation were added on or after the $3^{\text {rd }}$ week of vision therapy. ${ }^{27}$ The principles of substitution, adaptation, and habituation were applied to improve balance, postural control, dizziness, oscillopsia, and complaints of motion intolerance. ${ }^{30}$ In addition, independent daily light cardiovascular activity for 20 minutes, consistent with the Buffalo Treadmill Test, ${ }^{3}$ was recommended for all patients who were greater than three days post-injury.

\section{STATISTICAL METHODS}

All statistics were run in Stata for Mac. Descriptive data were calculated including means and standard deviations, with counts presented with percentages. Non-parametric and parametric statistics were used as appropriate after assessment of normal distribution patterns. Effect sizes were calculated with values of Cohen's $d$ considered large if $\geqslant 0.8$, medium if $0.5 \leqslant d<0.8$, and small if $0.2 \leqslant d<0.5$. Paired $t-$ tests were run using Bonferroni correction to control for familywise error and guard against false positives due to multiple comparisons $(\mathrm{p}<0.05)$.

\section{RESULTS}

The 38 patients with follow-up data received an average of $10.4 \pm 4.8$ treatment sessions over $57.6 \pm 34.0$ days. The average number of sessions addressing cervical dysfunction was $4.0 \pm 3.8$, vision and vestibular dysfunction $4.8 \pm 3.0$, and combined symptoms $1.7 \pm 0.8$.

Significant improvements were observed at post-test for general post-concussion symptoms on the patient-reported PCSS ( $\mathrm{p}=0.001, \mathrm{~d}=1.18$ ). See Table 2. Musculoskeletal, vi- sual, and vestibular symptom outcome measures improved as indicated on clinical measures and patient reported outcomes. Cervical ROM returned to normal for 15 of the 27 people who had limitations on evaluation (55.6\%). Vision function significantly improved for convergence on Brock string score $(p<0.001, d=1.20)$. Subjective vision symptoms assessed with the CISS also improved significantly $(\mathrm{p}<0.002$, $\mathrm{d}=1.04$ ). Divergence and abnormal saccades did not change significantly $(\mathrm{p}>0.05)$. Symptoms of BPPV were fully resolved for all 28/28 (100\%) diagnosed with BPPV. Vestibular system impairment represented by clinical measure of balance ability using the BESS improved significantly $(\mathrm{p}<0.001$, $\mathrm{d}=0.99$ ). The subjective patient-reported outcome $A B C$ scale also improved significantly post treatment $(\mathrm{p}<0.024$, $\mathrm{d}=-0.89$ ). Vestibulo-oculomotor symptoms improved significantly post-treatment, assessed with the DHI $(p<0.001$, $\mathrm{d}=1.04)$ and BIVSS $(\mathrm{p}<0.001, \mathrm{~d}=1.21)$. See Table 2.

\section{DISCUSSION}

The results of this retrospective cohort study showed that patients receiving physical therapy, consisting of manual therapy and exercise for cervical dysfunction and BPPV in the first three weeks post-concussion followed by vision and vestibular rehabilitation therapy beyond three weeks post injury, demonstrated clinical and patient-reported benefits in multiple systems. Improved cervical dysfunction such as limited ROM and headache following head injury, as well as other causes of post-concussion symptoms, can be contributed to by concomitant whiplash injury suffered at the time of the concussion. ${ }^{16}$ Changes in neck muscle activity during cervical rotation and cervico-ocular coordination may underlie clinical symptoms reported by people with visual deficits and changes in function, such as postural control, during cervical rotation. ${ }^{17}$ Regardless, whether cervicogenic headaches include pain, dizziness, and/or oculomotor dysfunction, ${ }^{8}$ restoring cervical ROM may well be one factor affecting multiple systems. ${ }^{31}$ Findings in the current study for the musculoskeletal, vision, and vestibular systems were consistent with past literature for select recommendation of the most recent clinical practice guidelines. ${ }^{10,11}$ 
Reducing musculoskeletal limitation was an important early goal because of the importance of cervical ROM for vision and vestibular assessments and rehabilitation and the impact cervical dysfunction has on headaches, visual and vestibular function. Most subjects regained full cervical ROM by re-evaluation, though data for some patients was missing, the measurement method was unreported, and the definition for full ROM can vary. ${ }^{7}$ Direct comparison of the study results with past reports that have shown that manual therapies improve cervical ROM with thrust or mobilization with movement ${ }^{31,32}$ was not possible because the retrospective data did not include specific manual techniques. Evidence for the efficacy of using manual therapy to reduce cervicogenic headache remains limited by the number of studies and lack of no-treatment control groups. ${ }^{8}$ One randomized controlled trial demonstrated increased ROM after sustained natural apophyseal glides but no significant improvement in dizziness or balance for people with chronic cervicogenic dizziness. ${ }^{31}$ Cervicogenic headache was not analyzed directly in this study, though headache was an item included in the total PCSS scores which improved significantly at re-evaluation. Starting care by addressing cervical dysfunction within the first three-weeks post-injury when indicated may be one among many factors in the improvements seen for cervical dysfunction as well as vision and vestibular outcome measures.

One common vision system dysfunction, convergence insufficiency, has been associated with other neurocognitive impairments and higher PCSS. ${ }^{33}$ After the study treatment, patient-reported symptoms on the PCSS had improved significantly as did clinical Brock string measures for near point convergence consistent with a five subject pilot study ${ }^{34}$ and a case-control study including 15 people with mild traumatic brain injury. ${ }^{35}$ Randomized controlled trials would be the next step to determine effect of vision therapy post-concussion compared to no treatment. Clinical measure of abnormal saccades did not change for patients posttreatment, consistent with past research. ${ }^{35}$ Missing data precluded analysis of other visual deficits common after minor traumatic brain injury such as smooth pursuits, and eye alignment. ${ }^{16}$ Also consistent with previous findings, patient-reported outcomes for vision symptoms improved significantly on the CISS and BIVSS. ${ }^{25,34,36}$ The oculomotor and vestibular systems are highly interconnected with integrated roles in maintaining balance, postural control and gaze stabilization, ${ }^{16}$ and patients exhibited benefits in both systems.

Evidence supports vestibular system rehabilitation for both central and peripheral deficits in concussion management. ${ }^{37}$ Physical therapy for cervical dysfunction has improved both musculoskeletal and vestibular symptoms including dizziness attributed to BPPV. ${ }^{37}$ In the current study, treatment for cervical dysfunction followed by canalith reposition procedures for BPPV was provided when indicated within the 3-week post injury timeframe instead of waiting for the 3-week post injury period when vision and vestibular rehabilitation began. Addressing BPPV before other vestibular deficits was similar to one past study, that aimed to reduce the confounding effect of BPPV on balance outcomes by treating BPPV first before introducing any habituation approaches. ${ }^{38}$ That all patients with BPPV had complete symptom resolution after treatment was comparable to past outcomes for the Epley maneuver summarized in a recent systematic review. ${ }^{39}$ Patient-reported outcome measures including the $\mathrm{ABC}$ scale and $\mathrm{DHI}$ both improved, consistent with a past retrospective cohort study of vestibular rehabilitation post-concussion. ${ }^{30}$

The recent clinical practice guidelines for mild traumatic brain injury supports multi-modal rehabilitation ${ }^{10,11}$ and physical therapy models of care for protracted concussion recovery have included cervical dysfunction and vestibularocular care and exertional activity without specific intervention sequence. ${ }^{40}$ The results of this study suggest that the intervention sequence ${ }^{27}$ addressing cervical dysfunction and BPPV within the three weeks post-injury before initiating vision and vestibular rehabilitation at more than three weeks post-injury may yield positive outcomes. Early cervical ROM restoration is important because cervical motion can have a confounding impact of cervicogenic headaches, and vision and vestibular function; while BPPV symptoms can confound both vestibular-ocular and vestibular spinal function including balance assessments. ${ }^{38}$ Since post-concussion symptoms resolve spontaneously for $80-90 \%$ of adults within 2 weeks, delaying therapeutic vision and vestibular training in the early period of recovery can avoid unnecessary symptom exacerbation. ${ }^{3}$ Within an overall integrated treatment approach, sequencing cervical dysfunction and BPPV treatment before advancing to vision and vestibular rehabilitation acknowledges the role cervical ROM plays in both vision and vestibular function $16,17,33,40$ and may improve the ability to perform functional activities early in the episode of care. Developing exercise tolerance thereafter would precede a return to sport. ${ }^{40}$ In total, the inter-relationships among the musculoskeletal, vision and vestibular systems, with respect to both function and treatment, make screening for and addressing cervical dysfunction a logical first step even before the three-week postinjury timeframe for starting vision and vestibular rehabilitation.

Limitations of this retrospective cohort analysis of deidentified preexisting data included the lack of a control group and inability to obtain follow-up or missing data. Specific sport involvement was not consistently recorded, although at least 10 concussions were due to skiing; and return to sport outcomes were not available. Headache pain, which was incorporated in the PCSS, was also not individually assessed. The small sample size limited ability to analyze sub-groups or perform regression analysis of factors associated with the outcomes. Patients with missing reevaluation data could not be included in the pre-post analysis, thus although effect size changes were large, results should be viewed with caution given unknown outcomes in at least $35.6 \%$. The potential exists for selection bias due to recruitment from a single concussion clinic. However, participation of the one concussion clinic allowed consistent use of the established protocol that sequenced and integrated musculoskeletal, vision and vestibular system care. Finally, the pragmatic treatment approach meant that variation in specific patient treatments was not controlled for, though results suggest that direct comparison of the study approach with a no-treatment control or alternate treatment condition would be warranted. 


\section{CONCLUSION}

After concussion, symptoms can arise from multiple systems with musculoskeletal, visual, and vestibular dysfunction. Because cervical ROM is a part of both the assessment and treatment of visual and vestibular dysfunction, cervical limitations can confound symptoms in both systems. The results of this study showed that patients with post-concussion symptoms receiving treatment sequenced to address cervical ROM limitations and BPPV symptoms in the first three weeks, integrated into visual and vestibular rehabilitation improved clinical and patient-reported outcomes for all systems. The interconnectedness of the systems makes sequencing care to address musculoskeletal function before the 3 -week post-concussion recovery window in a clinical approach that then integrates vision and vestibular system function a promising approach for concussion recovery.

\section{CONFLICTS OF INTEREST}

The authors have no conflicts to disclose.

\section{DISCLOSURES}

This study received no funding.

Submitted: February 29, 2020 CDT, Accepted: July 24, 2021 CDT 


\section{REFERENCES}

1. Langlois JA, Rutland-Brown W, Wald MM. The epidemiology and impact of traumatic brain injury. $J$ Head Trauma Rehabil. 2006;21(5):375-378. doi:10.109 7/00001199-200609000-00001

2. Herring SA, Cantu RC, Guskiewicz KM, et al. Concussion (mild traumatic brain injury) and the team physician: A consensus statement-2011 update. Med Sci Sports Exerc. 2011;43(12):2412-2422. doi:10.1 249/mss.0b013e3182342e64

3. Leddy JJ, Baker JG, Willer B. Active rehabilitation of concussion and post-concussion syndrome. Phys Med Rehabil Clin N Am. 2016;27(2):437-454. doi:10.1016/ j.pmr.2015.12.003

4. Schneider KJ, Leddy JJ, Guskiewicz KM, et al. Rest and treatment/rehabilitation following sport-related concussion: A systematic review. Br J Sports Med. 2017;51(12):930-934. doi:10.1136/bjsports-2016-0974 $\underline{75}$

5. Kostyun RO, Hafeez I. Protracted recovery from a concussion: A focus on gender and treatment interventions in an adolescent population. Sports Health. 2015;7(1):52-57. doi:10.1177/19417381145550 $\underline{75}$

6. Leslie O, Craton N. Concussion: Purely a brain injury? Clin J Sport Med. 2013;23(5):331-332. doi:10.1 097/jsm.0b013e318295bbb1

7. Tiwari D, Goldberg A, Yorke A, Marchetti GF, Alsalaheen B. Characterization of cervical spine impairments in children and adolescents postconcussion. Intl J Sports Phys Ther. 2019;14(2):282-295. doi:10.26603/ijspt20190282

8. Chaibi A, Russell MB. Manual therapies for cervicogenic headache: A systematic review. $J$ Headache Pain. 2012;13(5):351-359. doi:10.1007/s101 94-012-0436-7

9. Storey EP, Wiebe DJ, D’Alonzo BA, et al. Vestibular rehabilitation is associated with visuovestibular improvement in pediatric concussion. J Neurol Phys Ther. 2018;42(3):134-141. doi:10.1097/npt.000000000 $\underline{0000228}$

10. Marshall S, Bayley M, McCullagh S, et al. Updated clinical practice guidelines for concussion/mild traumatic brain injury and persistent symptoms. Brain Inj. 2015;29(6):688-700. doi:10.3109/0269905 2.2015.1004755
11. Quatman-Yates CC, Hunter-Giordano A, Shimamura KK, et al. Physical therapy evaluation and treatment after concussion/mild traumatic brain injury. J Orthop Sports Phys Ther. 2020;50(4):CPG1-CPG73.

12. Varatharajan S, Ferguson B, Chrobak K, et al. Are non-invasive interventions effective for the management of headaches associated with neck pain? An update of the Bone and Joint Decade Task Force on Neck Pain and Its Associated Disorders by the Ontario Protocol for Traffic Injury Management (OPTIMa) Collaboration. Eur Spine J. 2016;25(7):1971-1999. doi:10.1007/s00586-016-437 $\underline{6-9}$

13. Luedtke K, Allers A, Schulte LH, May A. Efficacy of interventions used by physiotherapists for patients with headache and migraine-systematic review and meta-analysis. Cephalalgia. 2015;36(5):474-492. doi:1 $\underline{0.1177 / 0333102415597889}$

14. Thiagarajan P, Ciuffreda KJ. Effect of oculomotor rehabilitation on vergence responsivity in mild traumatic brain injury. J Rehabil Res Dev. 2013;50(9):1223-1240. doi:10.1682/jrrd.2012.12.0235

15. Alsalaheen BA, Whitney SL, Mucha A, Morris LO, Furman JM, Sparto PJ. Exercise prescription patterns in patients treated with vestibular rehabilitation after concussion. Physiother Res Int. 2012;18(2):100-108. d oi:10.1002/pri.1532

16. Treleaven J. Dizziness, unsteadiness, visual disturbances, and sensorimotor control in traumatic neck pain. J Orthop Sports Phys Ther. 2017;47(7):492-502. doi:10.2519/jospt.2017.7052

17. Bexander CSM, Hodges PW. Cervico-ocular coordination during neck rotation is distorted in people with whiplash-associated disorders. Exp Brain Res. 2012;217(1):67-77. doi:10.1007/s00221-011-297 3-8

18. Kim EH, Granger-Donetti B, Vicci VR, Alvarez TL. The relationship between phoria and the ratio of convergence peak velocity to divergence peak velocity. Invest Ophthalmol Vis Sci. 2010;51(8):4017-4027. doi:10.1167/iovs.09-4560

19. Mestre C, Otero C, Díaz-Doutón F, Gautier J, Pujol J. An automated and objective cover test to measure heterophoria. Maiello G, ed. PLoS ONE. 2018;13(11):e0206674. doi:10.1371/journal.pone.020 $\underline{6674}$ 
20. Heick JD, Bay C, Valovich McLeod TC. Evaluation of vertical and horizontal saccades using the developmental eye movement test compared to the King-Devick test. Intl J Sports Phys Ther. 2018;13(5):808-818. doi:10.26603/ijspt20180808

21. Murray N, Salvatore A, Powell D, Reed-Jones R. Reliability and validity evidence of multiple balance assessments in athletes with a concussion. J Athl Train. 2014;49(4):540-549. doi:10.4085/1062-6050-4 9.3.32

22. Merritt VC, Bradson ML, Meyer JE, Arnett PA. Evaluating the test-retest reliability of symptom indices associated with the ImPACT post-concussion symptom scale (PCSS). J Clin Exp Neuropsychol. 2018;40(4):377-388. doi:10.1080/13803395.2017.1353 $\underline{590}$

23. Treleaven J. Dizziness Handicap Inventory (DHI). Aust J Physiother. 2006;52(1):67. doi:10.1016/s0004-95 14(06)70070-8

24. Rouse M, Borsting E, Mitchell GL, et al. Validity of the convergence insufficiency symptom survey: a confirmatory study. Optom Vis Sci. 2009;86(4):357-363. doi:10.1097/opx.0b013e3181989 $\underline{252}$

25. Laukkanen H, Scheiman M, Hayes JR. Brain Injury Vision Symptom Survey (BIVSS) Questionnaire. Optom Vis Sci. 2017;94(1):43-50. doi:10.1097/opx.000 $\underline{0000000000940}$

26. Alsalaheen BA, Whitney SL, Marchetti GF, et al. Relationship between cognitive assessment and balance measures in adolescents referred for vestibular physical therapy after concussion. Clin J Sport Med. 2016;26(1):46-52. doi:10.1097/jsm.000000 0000000185

27. Ziaks L, Giardina R, Kloos A. Integration of vision and vestibular therapy for vestibulo-ocular postconcussion disorder: A case study. Int J Allied Health Sci Pract. 2019;17(3):Article 11.

28. Argaet EC, Bradshaw AP, Welgampola MS. Benign positional vertigo, its diagnosis, treatment and mimics. Clin Neurophysiol Pract. 2019;4:97-111. doi:1 0.1016/i.cnp.2019.03.001

29. Scheiman M. Understanding and Managing Vision Deficits: A Guide for Occupational Therapists. 2nd ed. Thorofare, NJ: Slack Inc; 2002.

30. Alsalaheen BA, Mucha A, Morris LO, et al. Vestibular rehabilitation for dizziness and balance disorders after concussion. J Neurol Phys Ther. 2010;34(2):87-93. doi:10.1097/npt.0b013e3181dde568
31. Reid SA, Callister R, Katekar MG, Rivett DA. Effects of cervical spine manual therapy on range of motion, head repositioning, and balance in participants with cervicogenic dizziness: a randomized controlled trial. Arch Phys Med Rehabil. 2014;95(9):1603-1612. doi:10.1016/j.apmr.2014.04.00 9

32. Dunning JR, Cleland JA, Waldrop MA, et al. Upper cervical and upper thoracic thrust manipulation versus nonthrust mobilization in patients with mechanical neck pain: A multicenter randomized clinical trial. J Orthop Sports Phys Ther. 2012;42(1):5-18. doi:10.2519/jospt.2012.3894

33. Pearce KL, Sufrinko A, Lau BC, Henry L, Collins MW, Kontos AP. Near point of convergence after a sport-related concussion: Measurement reliability and relationship to neurocognitive impairment and symptoms. Am J Sports Med. 2015;43(12):3055-3061. doi:10.1177/0363546515606430

34. Scheiman MM, Talasan H, Mitchell GL, Alvarez TL. Objective assessment of vergence after treatment of concussion-related CI: A pilot study. Optom Vis Sci. 2017;94(1):74-88. doi:10.1097/opx.000000000000093 $\underline{6}$

35. Matuseviciene G, Johansson J, Möller M, Godbolt AK, Pansell T, Deboussard CN. Longitudinal changes in oculomotor function in young adults with mild traumatic brain injury in Sweden: An exploratory prospective observational study. BMJ Open. 2018;8(2):e018734. doi:10.1136/bmjopen-2017-01873

36. Kontos AP, Collins MW, Holland CL, et al. Preliminary evidence for improvement in symptoms, cognitive, vestibular, and oculomotor outcomes following targeted intervention with chronic mTBI patients. Mil Med. 2018;183(suppl_1):333-338. doi:1 $\underline{0.1093 / \mathrm{milmed} / \mathrm{usx} 172}$

37. Schneider KJ, Meeuwisse WH, Nettel-Aguirre A, et al. Cervicovestibular rehabilitation in sport-related concussion: A randomised controlled trial. Br J Sports Med. 2014;48(17):1294-1298. doi:10.1136/bjsports-20 $\underline{13-093267}$

38. Gurley JM, Hujsak BD, Kelly JL. Vestibular rehabilitation following mild traumatic brain injury. NRE. 2013;32(3):519-528. doi:10.3233/NRE-130874

39. Hilton MP, Pinder DK. The Epley (canalith repositioning) manoeuvre for benign paroxysmal positional vertigo. Cochrane Database of Systematic Reviews. 2014;(12):Art. No.: CD003162. doi:10.1002/1 4651858.cd003162.pub3 
40. Lundblad M. A conceptual model for physical therapists treating athletes with protracted recovery following a concussion. Int J Sports Phys Ther.

2017;12(2):286-296. 